\title{
Advances and challenges in understanding histone demethylase biology
}

Radoslaw P Nowak ${ }^{1,2^{*}}$, Anthony Tumber ${ }^{1,3^{*}}$, Catrine Johansson ${ }^{2,4^{*}}$, Ka Hing Che ${ }^{1,2 \&}$, Paul Brennan ${ }^{1,3}$, Dafydd Owen ${ }^{5}$, and Udo Oppermann ${ }^{1,2}$

${ }^{1}$ Structural Genomics Consortium, University of Oxford, Headington, OX3 7DQ, UK

${ }^{2}$ Botnar Research Centre, NIHR Oxford Biomedical Research Unit, Oxford OX3 7LD, UK

${ }^{3}$ Target Discovery Institute, University of Oxford, OX3 7FZ, UK

${ }^{4}$ Department of Chemistry, University of Oxford, OX1 3TA, UK

${ }^{5}$ Pfizer Worldwide Medicinal Chemistry, 610 Main Street, Cambridge MA02139, USA

*these authors contributed equally

\& present address: Gurdon Institute, University of Cambridge CB2 1QN, UK 


\begin{abstract}
Within the last decade we have witnessed significant progress in the field of chromatin methylation, ranging from the discovery that chromatin methylation is reversible, to the identification of two classes of oxidative chromatin demethylases. Multiple genetic and cellular studies emphasize the role of members of the amine oxidase and 2-oxoglutarate oxygenase enzyme families involved in methyl-lysine in physiology and disease. Advances in understanding of the underlying biochemistry have resulted in development of first series of clinical inhibitors and tool compounds which continue to resolve and help understand the complex relationships between chromatin modification, control of gene expression and metabolic states.
\end{abstract}




\section{INTRODUCTION}

Covalent modifications of chromatin components such as histones and DNA play central roles in transcriptional regulation, genome stability, $\mathrm{X}$ chromosome inactivation and imprinting, and are considered as part of a mechanism of epigenetic regulation [1, 2]. Among the multitude of combinatorial histone residue modifications, comprising acetylation, phosphorylation, ubiquitination and others, the methylation of arginine and lysine residues in particular, emerged as critical regulator of chromatin functions [3-8] leading to the hypothesis of a "histone code" $[9,10]$.

Histone lysine demethylases are members of two distinct enzyme families - The discovery of the first histone lysine demethylase (referred to as KDM 1 [11], lysinespecific demethylase 1 (LSD1, KDM1A), was described in 2004 by Shi et al. [12], shortly followed by Schüle et al. [13] and established two members of the enzyme family of flavine adenine dinucleotide (FAD) dependent amine oxidases as epigenetic regulators (Figure 1). Members of this enzyme family oxidize a range of biogenic amine containing substrates such as neurotransmitters, e.g. dopamine by MAOA or MAOB enzymes, or polyamines such as spermidine, cadaverine or putrescine by other members (Fig $1 \mathrm{~A})$. Within this family, two members (KDM1A and KDM1B) have demonstrated activity towards $\mathrm{N} \varepsilon$-methyl-lysine residues in histone $3(\mathrm{H} 3)$, acting on mono- or dimethyl lysyl residues 4 or 9 (H3K4me1/2 or H3K9me1/2 by KDM1A, and H3K4me1/2 by KDM1B) $[14,15]$. Shortly after, the discovery of the larger family of Jumonji-type of histone demethylases by Zhang et al [16-19] and others [20-28] highlighted the 2-oxoglutarate dependent oxygenases (2-OGs) as important mediators of chromatin and DNA modifications. This family of histone demethylases with more than 20 identified members in humans, contains a conserved catalytic JmjC domain composed of a double stranded beta helical fold (DSBH), and distinct members of demethylases (KDM2-7 highlighted in Fig 1B) show activity towards various methylated states of $\mathrm{H} 3 \mathrm{~K} 4, \mathrm{H} 3 \mathrm{~K} 9, \mathrm{H} 3 \mathrm{~K} 27$ and $\mathrm{H} 3 \mathrm{~K} 36$ (Figure 1).

These landmark discoveries provided a paradigm shift in histone lysine methylation: previously, this modification was considered to be a stable, post-translational modification of largely unknown significance: a modification which could only be removed by histone exchange or "dilution" during DNA replication [2, 29]. The NE group of lysine can be methylated in 3 different states (mono-, di-, tri-) whereas 
arginine residues can be mono-methylated or modified as symmetric/asymmetric dimethylated forms. The methylation state, residue position and the existence of specific recognition domains ("readers'), in addition to levels of specific methyltransferases ("writers") and demethylases ("erasers") dictate distinct functional and biological outcomes- for example, trimethylation of lysine 4 of histone 3 (H3K4me3) constitutes a "permissive" mark found around most transcriptionally active genes, whereas trimethylation of residues $\mathrm{H} 3 \mathrm{~K} 27$ and $\mathrm{H} 3 \mathrm{~K} 9$ is often found in "silenced" or transcriptionally inactive chromatin regions. From these and other groundbreaking genetic studies it is now clear that histone lysine methylation indeed is a reversible, dynamic and regulated process of importance for chromatin function [2], however the contribution of demethylase activity towards a physiological outcome is often not resolved or yet understood.

For further reading about the roles of KDMs in human biology and disease, we refer to several recent overviews $[1,2,6,7,30,31]$. Instead, we will provide in the following review a brief account of the current chemistry efforts to develop inhibitors towards the different types of KDM enzymes. This attempt is fueled by the need to utilize selective chemical tools to understand the enzymatic role of demethylase activity. Moreover several lines of evidence suggest therapeutic utility of demethylase inhibitors in a variety of human pathologies, at present largely pursued in oncology and inflammation. We further discuss metabolic implications of demethylase activity under different physiological states, and then turn attention to the issue of substrate specificity and the role of demethylases within larger chromatin protein complexes.

The catalytic mechanism of KDMs - Amine oxidation catalyzed by flavin-containing amine oxidases is characterized by oxidation of the $\alpha$-carbon bond of the substrate to form an imine intermediate, which, in turn, is hydrolyzed to form an unstable hemi-aminal that fragments into formaldehyde and the remaining lysylamine via a non-enzymatic process. In a complete catalytic cycle, the cofactor FAD is reduced to $\mathrm{FADH}_{2}$ and then is re-oxidized to produce hydrogen peroxide as byproduct $[12,32]$. This mechanism requires a protonated nitrogen atom, explaining the selectivity of amine oxidase -type KDM1A and B towards mono- and dimethylated lysyl residues (Figure 1C, Figure 2).

The human 2-OG oxygenase family consists of over 60 different members, with a 
significant proportion of bona fide histone demethylases (highlighted in Figure 1B), however the substrate spectrum of several members of this type of versatile oxygenases is not restricted to histone residues, and includes for example intermediary metabolites, nucleic acids, or amino acids residues in different proteins (such as prolyl and asparaginyl residues in matrix proteins (collagen) or transcription factors $(\mathrm{HIF} 1 \alpha))[30]$. The discovery that distinct members of this family, namely the TET (ten-eleven translocation) oxygenases are critically involved in the demethylation process of the epigenetic mark 5-methyl-cytosine in DNA, opened new avenues in understanding the significance of this chromatin modification [33-35]. Moreover, the discovery of $\mathrm{N}^{6}$-methyladenine in RNA and DNA, and oxidative reversal of this modification by 2-OG enzymes (such as ABH1, ABH5 or FTO, Figure 1) $[36,37]$ suggests that reversible nucleic acid modifications are more widespread than previously anticipated and also extends this principle into RNA biology.

Whereas the catalytic domain of the amine oxidases contains the typical $\alpha / \beta$ folding pattern typical of several types of oxidoreductases (and includes additional elements like the Tower domain found in KDM1A), the catalytic domain of JmjC- type oxygenases contains a highly conserved double- stranded $\beta$-helix (DSBH) fold with eight $\beta$-strands, also referred to as cupin, 'jelly-roll' or 'beta barrel' fold (Figure 2AB). In humans, members of this family share few conserved sequence motifs necessary for iron and cofactor binding, and are mechanistically defined by their ability to hydroxylate their specific substrates using a reactive oxo-ferryl (IV) intermediate (Figure 1D). In brief, the catalytic sequence of methyl-lysine demethylation proceeds through distinct steps of 2-OG cofactor and molecular oxygen binding and activation, resulting in a reactive oxo-ferryl (IV) intermediate that reacts with the specific substrate atom, usually resulting in a hydroxylated substrate and concomitant $\mathrm{CO}_{2}$ and succinate formation (Figure 1D, and Figure 2)[38, 39]. As in the case of amine oxidases, the hydroxylated substrate is an unstable hemi-aminal intermediate derived from the lysine methyl hydroxylation of the $\mathrm{N} \varepsilon$ - side-chain which then fragments into formaldehyde and a demethylated lysine side-chain. Importantly, this mechanism allows for demethylation of all possible Ne-methyl states (mono-, di- and tri-) found in methylated histone lysine residues and thus differs from the mechanism of monoamine oxidases. 
Are histone demethylases druggable therapeutic targets? Ample genetic and experimental evidence has provided the rationale for several inhibitor development programs, both in academia and the private sector, with advancements into several clinical trials for KDM1 inhibitors [40]. Since KDM chemotype development has been the subject of several recent comprehensive reviews $[38,41]$ we will here only briefly touch upon these efforts. With regards to KDM1 inhibitors, most programs have focused on mechanism-based inhibitors using the cyclopropylamine template tranylcypromine or derivatives such as GSK-LSD1 (Figure 3) which form covalent FAD adducts within the KDM1 active site (Figure 2E), however reversible inhibitors such as GSK354 have also been reported. Clearly, high-quality chemical tools will aid in dissecting KDM biology, however development of required potent, selective, and cell-active molecules for 2-OG KDMs has been challenging thus far. With most chemical matter occupying part of the 2-OG binding site, chemotypes have tended to be polar expressions of an iron-chelating pharmacophore (Figure 2), which combines to present physicochemical properties incompatible with cell penetration and is often secured through the introduction of a pro-drug. Given the high levels of 2-OG and other TCA-cycle derived metabolites within the cell, any inhibitors designed to compete for cofactor binding, need to show high affinity to achieve sufficient ontarget activity, as suggested through a recent chemoproteomics approach [42]. Substrate-binding competitive inhibitors appear to be more elusive and the possibilities for allosteric sites remain unproven. However, structure determinations of several of the major clinically relevant subfamilies of KDMs have enabled a better understanding of active site details (for example see KDM6B, Figure 2F) that allows to progress towards the challenge of highly selective inhibitors [43-50]. Although potent inhibitors have been discovered for most KDM sub-families, there are few compounds that meet criteria for chemical probes (Figure 3; see references [44, 47$49,51-62]$ ) due to a lack of selectivity and high potency in cells [63]. Taken together, there remains plenty of opportunity for the introduction of novel chemical probes for the demethylase family of enzymes.

Metabolic regulation of $\mathrm{KDMS}$ and epigenomic methylation signatures - the relationship between cellular metabolic states and chromatin modification is critical for understanding the long-term effects of transcriptional regulation, and as a consequence, the resulting cellular phenotypes. Chromatin modifying enzymes, i.e. 
methyl- or acetyl- transferases as well as demethylases or deacetylases (i.e. class II HDACs, sirtuins) utilise as cofactors metabolic intermediates such as Sadenosylmethione (SAM), acetyl-CoA, 2-oxoglutarate or NAD+, respectively, whose concentrations are subject to nutritional status and impact on global protein acetylation and methylation patterns [64]. For example, the critical role of 2oxoglutarate, and its impact on histone and DNA methylation with subsequent regulation of phenotypes has been recently demonstrated in pluripotency of stem cells [65] and in cancer [66]. Furthermore, several genetic studies highlight mutations in enzymes of the tricarboxylic acid (TCA) cycle such as fumarate hydratase, succinate dehydrogenase or isocitrate dehydrogenase (IDH) as drivers of malignant transformation and progression [66, 67]. Accordingly, the inhibitory effects of accumulated substrates (fumarate, succinate) or neomorphic products such as D-2hydroxyglutarate as in the case of IDH $[68,69]$, on chromatin hydroxylating KDM or TET enzymes [34], could explain the aberrant chromatin methylation patterns as cause for oncogenic drives. This is analogous to the inhibitory control exerted by succinate, observed during myeloid activation. Metabolic reprogramming induced by toll-like receptor (TLR) agonists such as lipopolysaccharide (LPS) derived from gram-negative bacteria increases succinate levels resulting in inhibition of the KDM related, 2OG oxygenase enzyme prolyl hydroxylase (PHD2), which controls the oxygen sensing transcription factor HIF1 $\alpha$. Importantly, this leads to up-regulation of HIF $1 \alpha$ target pathways under normoxic conditions [70, 71], with a concomitant expression of pro-inflammatory pathways during macrophage or dendritic cell activation. Oxygen levels themselves have a clear effect on global chromatin methylation states as observed during development and in several cancer types [7276]. This can be related to the fact that several demethylases such as members of KDM subfamilies 3-6 themselves are HIF1 $\alpha$ target genes [72, 76-82], however anaerobic metabolism can also increase concentrations of L-2-hydroxyglutarate via lactate or malate dehydrogenases, thereby inhibiting 2-OG enzymes such as KDMs. Changes in redox state could also affect susceptible amino acid residues, although the area of post-translational modifications of $\mathrm{KDMs}$ and resulting functional consequences requires further investigations. It is also conceivable that KDM enzyme activities (including LSD1/2, which are much less studied in this respect than 2-OG 
type KDMs) are directly limited by oxygen levels, leading to important alterations in chromatin methylation (Figure 4).

Beyond histone lysine methylation: what are the functions of demethylases? So far we have highlighted several points relating to the catalytic function of KDMs, their interrelated dependencies on cofactors such as oxygen and 2-OG, as well as inhibition by various metabolic intermediates. We assume that continued research will deliver novel insights into the relationships between metabolic control and chromatin methylation states. Another question is whether the catalytic function of KDMs is the sole or most important role in the context of chromatin biology [5]. Most, if not all histone demethylases are transiently associated to transcriptional regulators contributing to transcriptional activation or suppression [2]. In that respect, the importance of KDM scaffolding functions is highlighted in several instances (reviewed in [30]), and it is thought that KDMs create certain chromatin states, which provide a permissive environment for distinct transcriptional complexes. We believe that investigations into scaffolding functions of KDMs will be facilitated by genetic approaches, but also by specific and potent inhibitors, which will contribute to dissect specific roles of KDMs. We also predict that system-wide analysis of non-chromatin methylomes will deliver rich sets of possible novel KDM targets, thereby extending their substrate repertoire beyond chromatin biology. For example, a recent MS-based methylome study [83] revealed that $>500$ intracellular sites can be methylated, and multiple studies show the importance of this modification on their function, also suggesting that reversible methyl modifications could occur on non-histone proteins. For these reasons, the continued development and improvement of potent and specific KDM inhibitors will contribute to the understanding of chromatin biology, with the ultimate goal of understanding and treating human disease.

ACKNOWLEDGMENTS - Research in our laboratories is supported through funding from Arthritis Research UK (program grant number 20522), the NIHR Oxford Biomedical Research Unit, Cancer Research UK and the Rosetrees Trust. The Structural Genomics Consortium is a registered charity (number 1097737) that receives funds from Abbvie, Bayer Healthcare, Boehringer Ingelheim, the Canadian Institutes for Health Research, the Canadian Foundation for Innovation, Eli Lilly and Company, Genome Canada, GlaxoSmithKline, the Ontario Ministry of Economic 
Development and Innovation, Janssen, the Novartis Research Foundation, Pfizer, Takeda, and the Wellcome Trust.

\section{HIGHLIGHTS}

- histone methylation is critical in genome integrity and gene regulation

- histone lysine demethylation is accomplished by two distinct KDM enzyme families

- inhibitor development is ongoing including first clinical trials in oncology

- genetic and inhibitor approaches are needed to dissect the complex biology of KDMs

- cellular metabolic states control KDM activity and chromatin methylation

\section{REFERENCES}

1. Dawson, M.A., and Kouzarides, T. (2012). Cancer epigenetics: from mechanism to therapy. Cell 150, 12-27.

2. Kooistra, S.M., and Helin, K. (2012). Molecular mechanisms and potential functions of histone demethylases. Nat Rev Mol Cell Biol 13, 297-311.

3. Bannister, A.J., Schneider, R., and Kouzarides, T. (2002). Histone methylation: dynamic or static? Cell 109, 801-806.

4. Consortium, E.P. (2012). An integrated encyclopedia of DNA elements in the human genome. Nature 489, 57-74.

5. Ng, S.S., Yue, W.W., Oppermann, U., and Klose, R.J. (2009). Dynamic protein methylation in chromatin biology. Cell Mol Life Sci 66, 407-422.

6. Shi, Y., and Whetstine, J.R. (2007). Dynamic regulation of histone lysine methylation by demethylases. Mol Cell 25, 1-14.

7. Walport, L.J., Hopkinson, R.J., and Schofield, C.J. (2012). Mechanisms of human histone and nucleic acid demethylases. Curr Opin Chem Biol 16, 525-534.

8. Klose, R.J., and Zhang, Y. (2007). Regulation of histone methylation by demethylimination and demethylation. Nat Rev Mol Cell Biol 8, 307-318.

9. Jenuwein, T., and Allis, C.D. (2001). Translating the histone code. Science 293, 1074-1080.

10. Wang, Y., Fischle, W., Cheung, W., Jacobs, S., Khorasanizadeh, S., and Allis, C.D. (2004). Beyond the double helix: writing and reading the histone code. Novartis Found Symp 259, 3-17; discussion 17-21, 163-169.

11. Allis, C.D., Berger, S.L., Cote, J., Dent, S., Jenuwien, T., Kouzarides, T., Pillus, L., Reinberg, D., Shi, Y., Shiekhattar, R., et al. (2007). New nomenclature for chromatin-modifying enzymes. Cell 131, 633-636. 
12. Shi, Y., Lan, F., Matson, C., Mulligan, P., Whetstine, J.R., Cole, P.A., Casero, R.A., and Shi, Y. (2004). Histone demethylation mediated by the nuclear amine oxidase homolog LSD1. Cell 119, 941-953.

13. Metzger, E., Wissmann, M., Yin, N., Muller, J.M., Schneider, R., Peters, A.H., Gunther, T., Buettner, R., and Schule, R. (2005). LSD1 demethylates repressive histone marks to promote androgen-receptor-dependent transcription. Nature 437, 436-439.

14. Chen, F., Yang, H., Dong, Z., Fang, J., Wang, P., Zhu, T., Gong, W., Fang, R., Shi, Y.G., Li, Z., et al. (2013). Structural insight into substrate recognition by histone demethylase LSD2/KDM1b. Cell Res 23, 306-309.

15. Stavropoulos, P., Blobel, G., and Hoelz, A. (2006). Crystal structure and mechanism of human lysine-specific demethylase-1. Nat Struct Mol Biol 13, 626632.

16. Klose, R.J., Yamane, K., Bae, Y., Zhang, D., Erdjument-Bromage, H., Tempst, P., Wong, J., and Zhang, Y. (2006). The transcriptional repressor JHDM3A demethylates trimethyl histone H3 lysine 9 and lysine 36. Nature 442, 312-316.

17. Klose, R.J., Yan, Q., Tothova, Z., Yamane, K., Erdjument-Bromage, H., Tempst, P., Gilliland, D.G., Zhang, Y., and Kaelin, W.G., Jr. (2007). The retinoblastoma binding protein RBP2 is an H3K4 demethylase. Cell 128, 889-900.

18. Lee, N., Zhang, J., Klose, R.J., Erdjument-Bromage, H., Tempst, P., Jones, R.S., and Zhang, Y. (2007). The trithorax-group protein Lid is a histone H3 trimethyl-Lys4 demethylase. Nat Struct Mol Biol 14, 341-343.

19. Tsukada, Y., Fang, J., Erdjument-Bromage, H., Warren, M.E., Borchers, C.H., Tempst, P., and Zhang, Y. (2006). Histone demethylation by a family of JmjC domain-containing proteins. Nature 439, 811-816.

20. Christensen, J., Agger, K., Cloos, P.A., Pasini, D., Rose, S., Sennels, L., Rappsilber, J., Hansen, K.H., Salcini, A.E., and Helin, K. (2007). RBP2 belongs to a family of demethylases, specific for tri-and dimethylated lysine 4 on histone 3. Cell 128, 1063-1076.

21. Cloos, P.A., Christensen, J., Agger, K., Maiolica, A., Rappsilber, J., Antal, T., Hansen, K.H., and Helin, K. (2006). The putative oncogene GASC1 demethylates tri- and dimethylated lysine 9 on histone H3. Nature 442, 307-311.

22. De Santa, F., Totaro, M.G., Prosperini, E., Notarbartolo, S., Testa, G., and Natoli, G. (2007). The histone H3 lysine-27 demethylase Jmjd3 links inflammation to inhibition of polycomb-mediated gene silencing. Cell 130, 1083-1094.

23. Hong, S., Cho, Y.W., Yu, L.R., Yu, H., Veenstra, T.D., and Ge, K. (2007). Identification of JmjC domain-containing UTX and JMJD3 as histone H3 lysine 27 demethylases. Proc Natl Acad Sci U S A 104, 18439-18444.

24. Iwase, S., Lan, F., Bayliss, P., de la Torre-Ubieta, L., Huarte, M., Qi, H.H., Whetstine, J.R., Bonni, A., Roberts, T.M., and Shi, Y. (2007). The X-linked mental retardation gene SMCX/JARID1C defines a family of histone H3 lysine 4 demethylases. Cell $128,1077-1088$.

25. Lee, M.G., Norman, J., Shilatifard, A., and Shiekhattar, R. (2007). Physical and functional association of a trimethyl H3K4 demethylase and Ring6a/MBLR, a polycomb-like protein. Cell 128, 877-887.

26. Pasini, D., Hansen, K.H., Christensen, J., Agger, K., Cloos, P.A., and Helin, K. (2008). Coordinated regulation of transcriptional repression by the RBP2 H3K4 demethylase and Polycomb-Repressive Complex 2. Genes Dev 22, 1345-1355.

27. Seward, D.J., Cubberley, G., Kim, S., Schonewald, M., Zhang, L., Tripet, B., and Bentley, D.L. (2007). Demethylation of trimethylated histone H3 Lys4 in vivo by JARID1 JmjC proteins. Nat Struct Mol Biol 14, 240-242.

28. Tahiliani, M., Mei, P., Fang, R., Leonor, T., Rutenberg, M., Shimizu, F., Li, J., Rao, A., and Shi, Y. (2007). The histone H3K4 demethylase SMCX links REST target genes to X-linked mental retardation. Nature 447, 601-605. 
29. Paik, W.K., and Kim, S. (1973). Enzymatic demethylation of calf thymus histones. Biochem Biophys Res Commun 51, 781-788.

30. Johansson, C., Tumber, A., Che, K., Cain, P., Nowak, R., Gileadi, C., and Oppermann, U. (2014). The roles of Jumonji-type oxygenases in human disease. Epigenomics 6, 89-120.

31. Klose, R.J., Kallin, E.M., and Zhang, Y. (2006). JmjC-domain-containing proteins and histone demethylation. Nat Rev Genet 7, 715-727.

32. Binda, C., Mattevi, A., and Edmondson, D.E. (2002). Structure-function relationships in flavoenzyme-dependent amine oxidations: a comparison of polyamine oxidase and monoamine oxidase. J Biol Chem 277, 23973-23976.

33. Ito, S., Shen, L., Dai, Q., Wu, S.C., Collins, L.B., Swenberg, J.A., He, C., and Zhang, Y. (2011). Tet proteins can convert 5-methylcytosine to 5-formylcytosine and 5carboxylcytosine. Science 333, 1300-1303.

34. Shen, L., Song, C.X., He, C., and Zhang, Y. (2014). Mechanism and function of oxidative reversal of DNA and RNA methylation. Annu Rev Biochem 83, 585-614. This reference provides an overview on the role of oxygenases in mediating DNA and RNA demethylation

35. Tahiliani, M., Koh, K.P., Shen, Y., Pastor, W.A., Bandukwala, H., Brudno, Y., Agarwal, S., Iyer, L.M., Liu, D.R., Aravind, L., et al. (2009). Conversion of 5methylcytosine to 5-hydroxymethylcytosine in mammalian DNA by MLL partner TET1. Science 324, 930-935.

36. Wu, T.P., Wang, T., Seetin, M.G., Lai, Y., Zhu, S., Lin, K., Liu, Y., Byrum, S.D., Mackintosh, S.G., Zhong, M., et al. (2016). DNA methylation on N(6)-adenine in mammalian embryonic stem cells. Nature 532, 329-333. This discovery expands the repertoire of DNA methylation beyond cytosine modification.

37. Zheng, G., Dahl, J.A., Niu, Y., Fedorcsak, P., Huang, C.M., Li, C.J., Vagbo, C.B., Shi, Y., Wang, W.L., Song, S.H., et al. (2013). ALKBH5 is a mammalian RNA demethylase that impacts RNA metabolism and mouse fertility. Mol Cell 49, 18-29. This reference demonstrates the role of oxygenases in RNA demethylation.

38. McAllister, T.E., England, K.S., Hopkinson, R.J., Brennan, P.E., Kawamura, A., and Schofield, C.J. (2016). Recent Progress in Histone Demethylase Inhibitors. J Med Chem 59, 1308-1329.

39. McDonough, M.A., Loenarz, C., Chowdhury, R., Clifton, I.J., and Schofield, C.J. (2010). Structural studies on human 2-oxoglutarate dependent oxygenases. Curr Opin Struct Biol 20,659-672.

40. Mould, D.P., McGonagle, A.E., Wiseman, D.H., Williams, E.L., and Jordan, A.M. (2015). Reversible inhibitors of LSD1 as therapeutic agents in acute myeloid leukemia: clinical significance and progress to date. Med Res Rev 35, 586-618.

41. Maes, T., Carceller, E., Salas, J., Ortega, A., and Buesa, C. (2015). Advances in the development of histone lysine demethylase inhibitors. Curr Opin Pharmacol 23, 52-60.

42. Joberty, G., Boesche, M., Brown, J.A., Eberhard, D., Garton, N.S., Humphreys, P.G., Mathieson, T., Muelbaier, M., Ramsden, N.G., Reader, V., et al. (2016). Interrogating the Druggability of the 2-Oxoglutarate-Dependent Dioxygenase Target Class by Chemical Proteomics. ACS Chem Biol. This reference describes a novel chemoproteomics approach to assess the druggability of Jmj-type demethylases.

43. Johansson, C., Velupillai, S., Tumber, A., Szykowska, A., Hookway, E.S., Nowak, R.P., Strain-Damerell, C., Gileadi, C., Philpott, M., Burgess-Brown, N., et al. (2016). Structural analysis of human KDM5B guides histone demethylase inhibitor development. Nat Chem Biol. This reference together with reference 46 demonstrates development of selective and potent KDM chemical probes

44. Kruidenier, L., Chung, C.W., Cheng, Z., Liddle, J., Che, K., Joberty, G., Bantscheff, M., Bountra, C., Bridges, A., Diallo, H., et al. (2012). A selective jumonji H3K27 
demethylase inhibitor modulates the proinflammatory macrophage response. Nature 488, 404-408. This initial discovery of inhibitors for KDM6 demonstrated the druggability of 2-OG- type KDM enzymes.

45. Ng, S.S., Kavanagh, K.L., McDonough, M.A., Butler, D., Pilka, E.S., Lienard, B.M., Bray, J.E., Savitsky, P., Gileadi, O., von Delft, F., et al. (2007). Crystal structures of histone demethylase JMJD2A reveal basis for substrate specificity. Nature 448, 87-91.

46. Vinogradova, M., Gehling, V.S., Gustafson, A., Arora, S., Tindell, C.A., Wilson, C., Williamson, K.E., Guler, G.D., Gangurde, P., Manieri, W., et al. (2016). An inhibitor of KDM5 demethylases reduces survival of drug-tolerant cancer cells. Nat Chem Biol. This reference together with reference 43 demonstrates development of selective and potent KDM5 chemical probes

47. Westaway, S.M., Preston, A.G., Barker, M.D., Brown, F., Brown, J.A., Campbell, M., Chung, C.W., Diallo, H., Douault, C., Drewes, G., et al. (2016). Cell Penetrant Inhibitors of the KDM4 and KDM5 Families of Histone Lysine Demethylases. 1. 3Amino-4-pyridine Carboxylate Derivatives. J Med Chem 59, 1357-1369.

48. Westaway, S.M., Preston, A.G., Barker, M.D., Brown, F., Brown, J.A., Campbell, M., Chung, C.W., Drewes, G., Eagle, R., Garton, N., et al. (2016). Cell Penetrant Inhibitors of the KDM4 and KDM5 Families of Histone Lysine Demethylases. 2. Pyrido[3,4-d]pyrimidin-4(3H)-one Derivatives. J Med Chem.

49. Heinemann, B., Nielsen, J.M., Hudlebusch, H.R., Lees, M.J., Larsen, D.V., Boesen, T., Labelle, M., Gerlach, L.O., Birk, P., and Helin, K. (2014). Inhibition of demethylases by GSK-J1/J4. Nature 514, E1-2.

50. Hillringhaus, L., Yue, W.W., Rose, N.R., Ng, S.S., Gileadi, C., Loenarz, C., Bello, S.H., Bray, J.E., Schofield, C.J., and Oppermann, U. (2011). Structural and evolutionary basis for the dual substrate selectivity of human KDM4 histone demethylase family. J Biol Chem 286, 41616-41625.

51. Bavetsias, V., Lanigan, R.M., Ruda, G.F., Atrash, B., McLaughlin, M.G., Tumber, A., Mok, N.Y., Le Bihan, Y.V., Dempster, S., Boxall, K.J., et al. (2016). 8-Substituted Pyrido[3,4-d]pyrimidin-4(3H)-one Derivatives As Potent, Cell Permeable, KDM4 (JMJD2) and KDM5 (JARID1) Histone Lysine Demethylase Inhibitors. J Med Chem 59, 1388-1409.

52. Dhanak, D. (2013). Drugging the cancer genome. Proceedings of the 104th Annual Meeting of the Ammerican Association for Cancer Research.

53. England, K.S., Tumber, A., Krojer, T., Scozzafava, G., Ng, S.S., Daniel, M., Szykowska, A., Che, K., von Delft, F., Burgess-Brown, N.A., et al. (2014). Optimisation of a triazolopyridine based histone demethylase inhibitor yields a potent and selective KDM2A (FBXL11) inhibitor. Medchemcomm 5, 1879-1886.

54. Hopkinson, R.J., Tumber, A., Yapp, C., Chowdhury, R., Aik, W., Che, K.H., Li, X.S., Kristensen, J.B., King, O.N., Chan, M.C., et al. (2013). 5-Carboxy-8hydroxyquinoline is a Broad Spectrum 2-Oxoglutarate Oxygenase Inhibitor which Causes Iron Translocation. Chem Sci 4, 3110-3117.

55. Hu, J., Wang, X., Chen, L., Huang, M., Tang, W., Zuo, J., Liu, Y.C., Shi, Z., Liu, R., Shen, J., et al. (2015). Design and discovery of new pyrimidine coupled nitroaromatic rings as chelating groups of JMJD3 inhibitors. Bioorganic Med Chem Lett. 26, 721-725.

56. Albrecht, B.K., Gehling, V.S., Harmange, J.C., Lai, T., Liang, J., Dragovich, P., Ortwine, D., Labadie, S., Zhang, B., and Kiefer, J. (2015). Therapeutic compounds and uses thereof. W02015135094 A1.

57. Labelle, M., Boehsen, T., Mehrotra, M., Khan, Q., and Ullah, F. (2011). Inhibitors of Demethylases. W02014053491 A1.

58. Luo, X., Liu, Y., Kubicek, S., Myllyharju, J., Tumber, A., Ng, S., Che, K.H., Podoll, J., Heightman, T.D., Oppermann, U., et al. (2011). A selective inhibitor and probe of 
the cellular functions of Jumonji C domain-containing histone demethylases. J Am Chem Soc 133, 9451-9456.

59. Mohammad, H.P., Smitheman, K.N., Kamat, C.D., Soong, D., Federowicz, K.E., Van Aller, G.S., Schneck, J.L., Carson, J.D., Liu, Y., Butticello, M., et al. (2015). A DNA Hypomethylation Signature Predicts Antitumor Activity of LSD1 Inhibitors in SCLC. Cancer Cell 28, 57-69. This reference shows the druggability of KDM1 enzymes.

60. Rose, N.R., Woon, E.C., Tumber, A., Walport, L.J., Chowdhury, R., Li, X.S., King, O.N., Lejeune, C., Ng, S.S., Krojer, T., et al. (2012). Plant growth regulator daminozide is a selective inhibitor of human KDM2/7 histone demethylases. J Med Chem 55, 6639-6643.

61. Suzuki, T., Ozasa, H., Itoh, Y., Zhan, P., Sawada, H., Mino, K., Walport, L., Ohkubo, R., Kawamura, A., Yonezawa, M., et al. (2013). Identification of the KDM2/7 histone lysine demethylase subfamily inhibitor and its antiproliferative activity. J Med Chem 56, 7222-7231.

62. Upadhyay, A.K., Rotili, D., Han, J.W., Hu, R., Chang, Y., Labella, D., Zhang, X., Yoon, Y.S., Mai, A., and Cheng, X. (2012). An analog of BIX-01294 selectively inhibits a family of histone $\mathrm{H} 3$ lysine 9 Jumonji demethylases. J Mol Biol 416, 319-327.

63. Frye, S.V. (2010). The art of the chemical probe. Nat Chem Biol 6, 159-161.

64. Su, X., Wellen, K.E., and Rabinowitz, J.D. (2016). Metabolic control of methylation and acetylation. Curr Opin Chem Biol 30, 52-60.

65. Carey, B.W., Finley, L.W., Cross, J.R., Allis, C.D., and Thompson, C.B. (2015). Intracellular alpha-ketoglutarate maintains the pluripotency of embryonic stem cells. Nature 518, 413-416.

66. Xiao, M., Yang, H., Xu, W., Ma, S., Lin, H., Zhu, H., Liu, L., Liu, Y., Yang, C., Xu, Y., et al. (2012). Inhibition of alpha-KG-dependent histone and DNA demethylases by fumarate and succinate that are accumulated in mutations of FH and SDH tumor suppressors. Genes Dev 26, 1326-1338.

67. Letouze, E., Martinelli, C., Loriot, C., Burnichon, N., Abermil, N., Ottolenghi, C., Janin, M., Menara, M., Nguyen, A.T., Benit, P., et al. (2013). SDH mutations establish a hypermethylator phenotype in paraganglioma. Cancer Cell 23, 739752.

68. Chowdhury, R., Yeoh, K.K., Tian, Y.M., Hillringhaus, L., Bagg, E.A., Rose, N.R., Leung, I.K., Li, X.S., Woon, E.C., Yang, M., et al. (2011). The oncometabolite 2hydroxyglutarate inhibits histone lysine demethylases. EMBO Rep 12, 463-469.

69. Losman, J.A., and Kaelin, W.G., Jr. (2013). What a difference a hydroxyl makes: mutant IDH, (R)-2-hydroxyglutarate, and cancer. Genes Dev 27, 836-852.

70. Kelly, B., and O'Neill, L.A. (2015). Metabolic reprogramming in macrophages and dendritic cells in innate immunity. Cell Res 25, 771-784.

71. Tannahill, G.M., Curtis, A.M., Adamik, J., Palsson-McDermott, E.M., McGettrick, A.F., Goel, G., Frezza, C., Bernard, N.J., Kelly, B., Foley, N.H., et al. (2013). Succinate is an inflammatory signal that induces IL-1beta through HIF-1alpha. Nature 496, 238-242.

72. Hancock, R.L., Dunne, K., Walport, L.J., Flashman, E., and Kawamura, A. (2015). Epigenetic regulation by histone demethylases in hypoxia. Epigenomics 7, 791811.

73. Johnson, A.B., Denko, N., and Barton, M.C. (2008). Hypoxia induces a novel signature of chromatin modifications and global repression of transcription. Mutat Res 640, 174-179.

74. Perez-Perri, J.I., Acevedo, J.M., and Wappner, P. (2011). Epigenetics: new questions on the response to hypoxia. Int J Mol Sci 12, 4705-4721.

75. Tausendschon, M., Dehne, N., and Brune, B. (2011). Hypoxia causes epigenetic gene regulation in macrophages by attenuating Jumonji histone demethylase activity. Cytokine 53, 256-262. 
76. Xia, X., Lemieux, M.E., Li, W., Carroll, J.S., Brown, M., Liu, X.S., and Kung, A.L. (2009). Integrative analysis of HIF binding and transactivation reveals its role in maintaining histone methylation homeostasis. Proc Natl Acad Sci U S A 106, 4260-4265.

77. Beyer, S., Kristensen, M.M., Jensen, K.S., Johansen, J.V., and Staller, P. (2008). The histone demethylases JMJD1A and JMJD2B are transcriptional targets of hypoxia-inducible factor HIF. J Biol Chem 283, 36542-36552.

78. Lee, H.Y., Choi, K., Oh, H., Park, Y.K., and Park, H. (2014). HIF-1-dependent induction of Jumonji domain-containing protein (JMJD) 3 under hypoxic conditions. Mol Cells 37, 43-50.

79. Niu, X., Zhang, T., Liao, L., Zhou, L., Lindner, D.J., Zhou, M., Rini, B., Yan, Q., and Yang, H. (2012). The von Hippel-Lindau tumor suppressor protein regulates gene expression and tumor growth through histone demethylase JARID1C. Oncogene 31, 776-786.

80. Pollard, P.J., Loenarz, C., Mole, D.R., McDonough, M.A., Gleadle, J.M., Schofield, C.J., and Ratcliffe, P.J. (2008). Regulation of Jumonji-domain-containing histone demethylases by hypoxia-inducible factor (HIF)-1alpha. Biochem J 416, 387-394.

81. Shmakova, A., Batie, M., Druker, J., and Rocha, S. (2014). Chromatin and oxygen sensing in the context of JmjC histone demethylases. Biochem J 462, 385-395.

82. Wellmann, S., Bettkober, M., Zelmer, A., Seeger, K., Faigle, M., Eltzschig, H.K., and Buhrer, C. (2008). Hypoxia upregulates the histone demethylase JMJD1A via HIF1. Biochem Biophys Res Commun 372, 892-897.

83. Cao, X.J., Arnaudo, A.M., and Garcia, B.A. (2013). Large-scale global identification of protein lysine methylation in vivo. Epigenetics $8,477-485$. This reference provides the first systematic approach to analyse the lysine-methylome. 


\section{LEGEND to FIGURES}

Figure 1: Histone demethylases belong to two distinct enzyme families. KDM1A and KDM1B are members of the amine oxidase family (A), utilize a FAD-dependent oxidase mechanism to generate a hemiaminal intermediate that fragments into a demethylated product and formaldehyde (C). Other histone demethylases are members of the 2-oxoglutarate oxygenase family with various identified subfamilies (KDM2-7), that utilize a Fe (IV)-oxoferryl species (generated by 2-oxoglutarate to succinate formation) to produce a hemiaminal species, leading to a demethylated residue and formaldehyde.

Figure 2: Structural overview of the two KDM enzyme families. Ribbon representation of A) amine oxidases (KDM1A, pdb 2UXN) and B) $20 \mathrm{O}$ dependent di-oxygenases (KDM4A, pdb 2OQ6) showing the catalytic oxidase- and JmjCdomain coloured in blue. Cofactor and peptide binding pocket in C) KDM1A (2UXN) and D) KDM4A (2OQ6). E) Inhibitor 1-ethyl-Tranylcypromine (D70) bound in the peptide binding pocket of KDM1A (4UV9) F) Inhibitor GSK-J1 bound to the cofactor binding pocket in KDM6B (4ASK).

\section{Figure 3: Overview on selected KDM inhibitor chemotypes.}

Figure 4: KDM enzymes influence epigenomic signatures via enzymatic and scaffolding functions. The enzymatic function can be regulated through several variables, including signals such as growth factors, cytokines, metabolic state, endogenous or xenobiotic inhibitors, and oxygen concentration. 
FIGURE 1

A
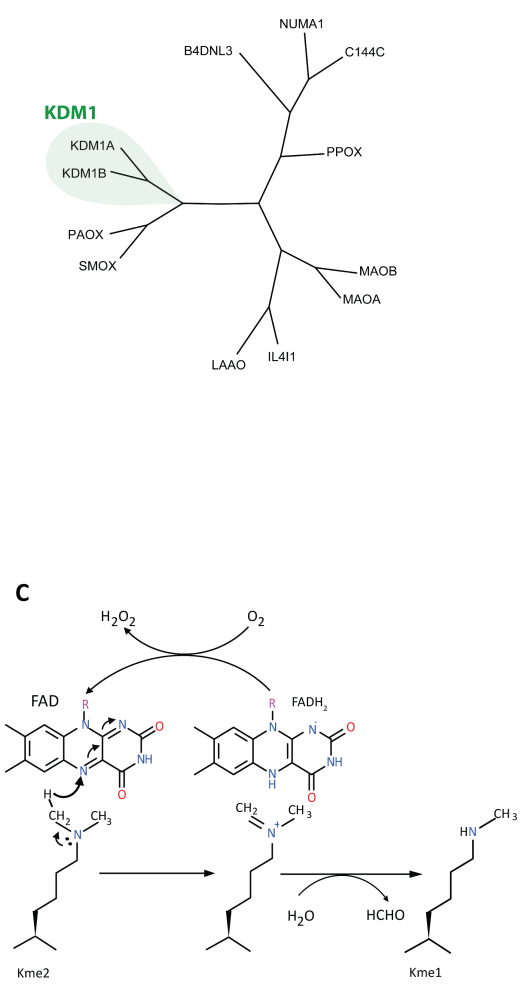
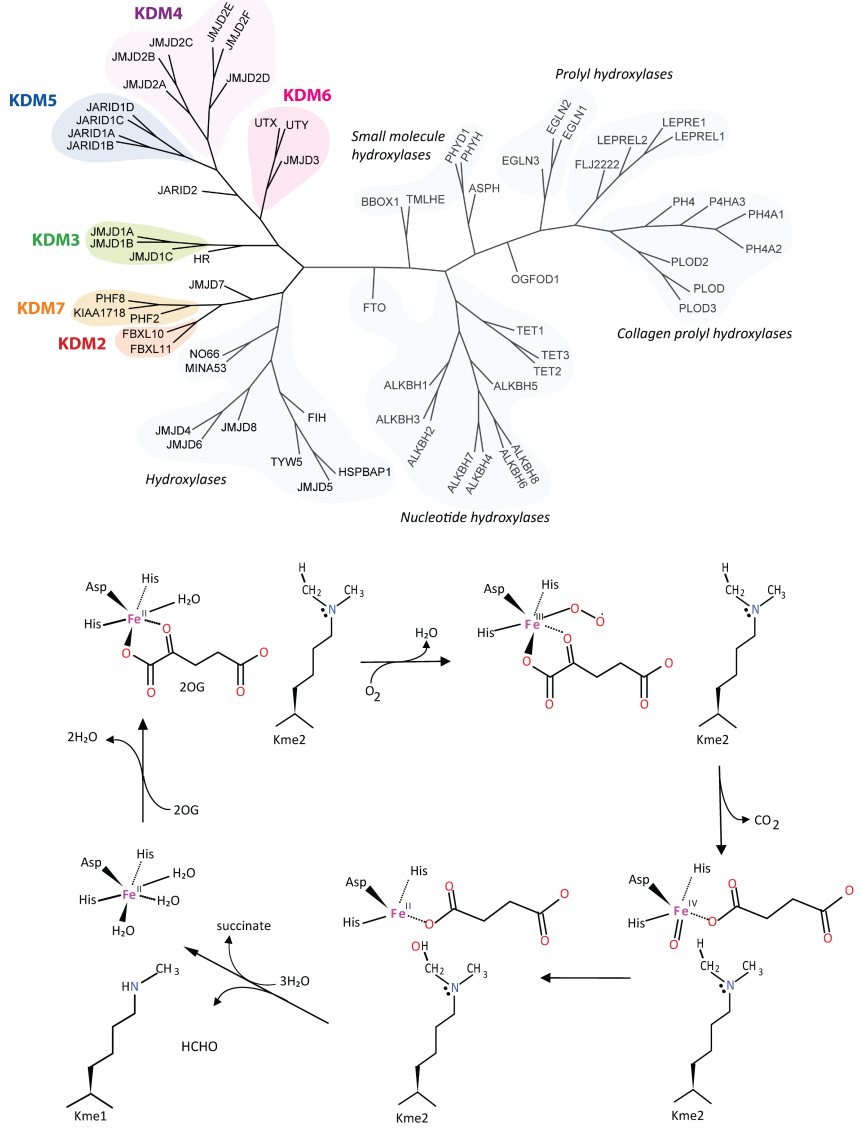


\section{FIGURE 2}

A

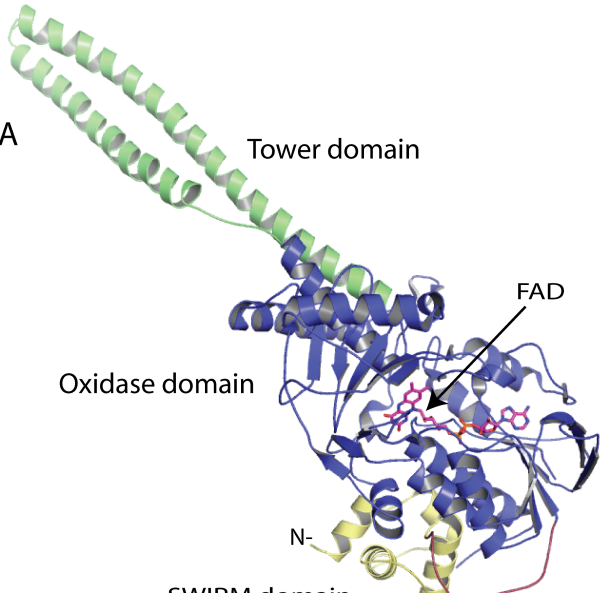

SWIRM domain

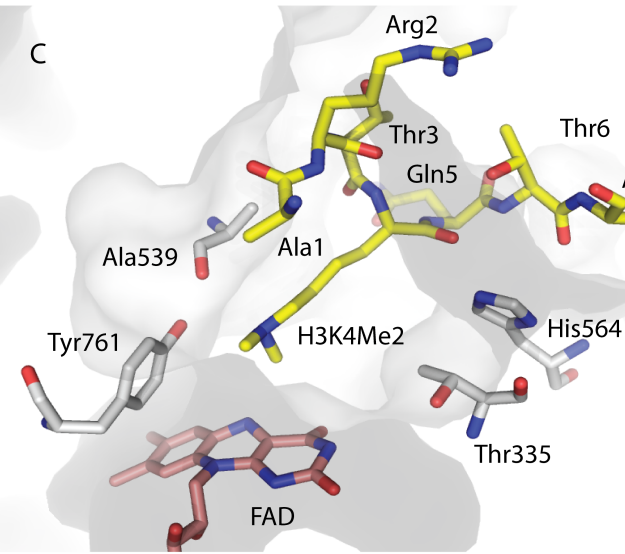

E

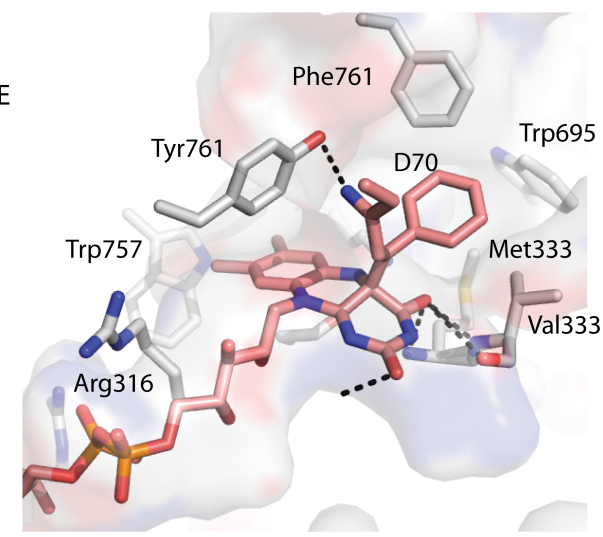

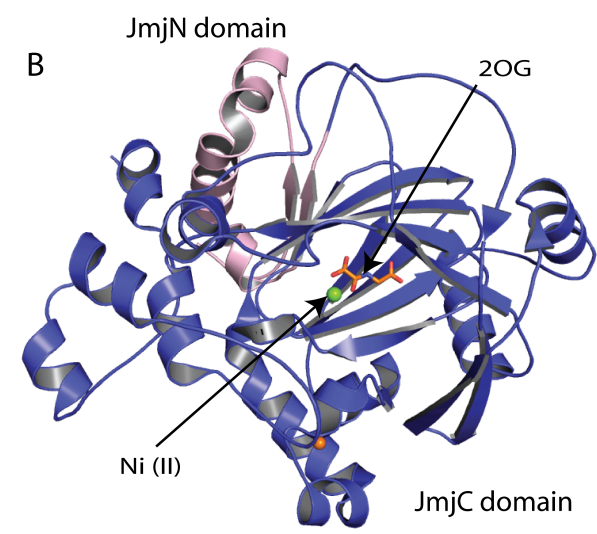

D
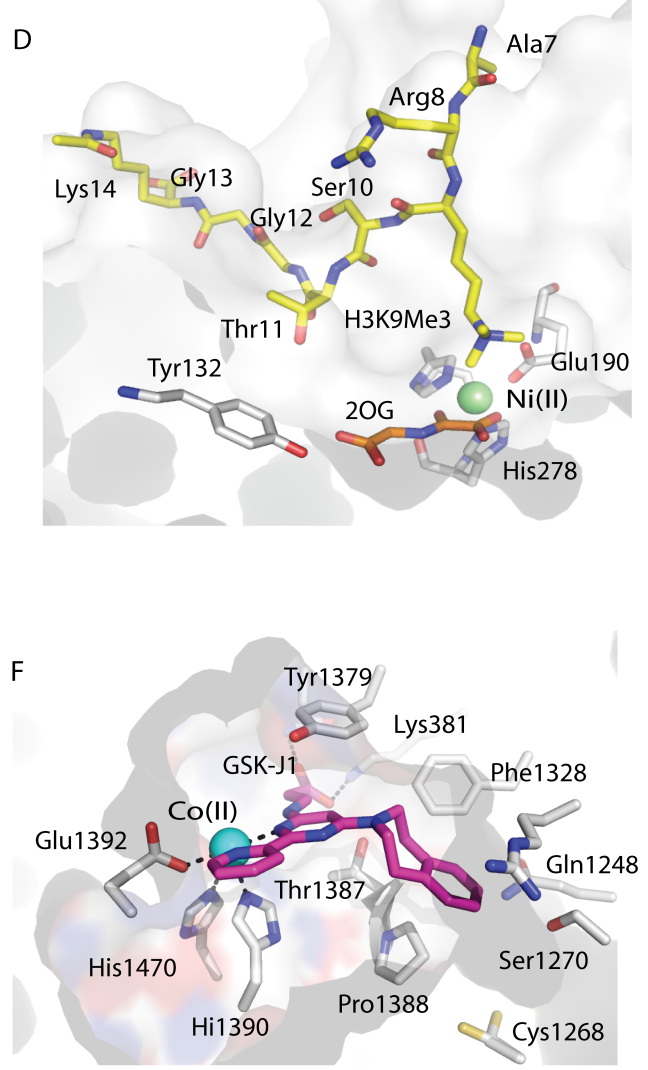
FIGURE 3

KDM1<smiles>CCCNc1ccccc1</smiles>

GSK-LSD 159
KDM1A K.PpP 0160 KDM1A $K_{i}^{\text {app }} 0.160 \mu \mathrm{M}$
sective over KDM1B, MAO-AIB

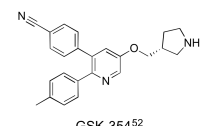

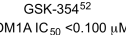
sDMTACtive over MAO-A $\mathrm{AB}$
sele
KDM2/7, PHF8

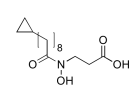

Cmpd 9 from 61

DM7B I I $501.2 \mu \mathrm{MM}$ KDM2A IC $50.8 \mu \mathrm{M}$

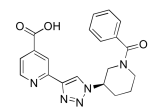

Cmpd 35 from ${ }^{53}$

KDM2A IC $50.063 \mu \mathrm{M}$

DM4A/4C IC $5016 \mu \mathrm{M}$

$$
\text { 等 }
$$

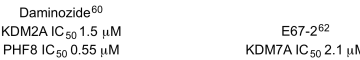

KDM3A4AE/6B IC SD $^{\circ} 100$ uM
KDM4/5

$$
\text { 每 }
$$

Cmpd 39 from 47
KDM4A/4C/4D/4E/5C IC S $_{50} 0.04-0.1 \mu \mathrm{M}$
KDM6B IC $50.13 \mu \mathrm{M}$

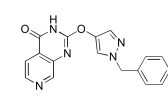

Cmpd 41 from 48
KDM4A/4C/4D/4E 1 I $_{50} 0.40-0.63 \mu \mathrm{M}$ KDM5C IC ${ }_{50} 0.06 \mu \mathrm{M}$
$\mathrm{KDM} 6 \mathrm{~B}, \mathrm{PHD} 3 \mathrm{IC}_{50}>100 \mu \mathrm{M}$

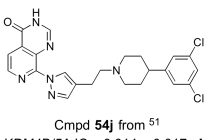

Cmpd 54 f from ${ }^{51}$
KDM4B/5A IC $50.014-0.017$ KDM4B/5A I I $50.014-0.017 \mu \mathrm{MM}$ KDM2A I I $5_{50} 2.4 \mu \mathrm{M}$
KDM3A $\mathrm{IC}_{50} 3.1 \mu \mathrm{M}$ KDM6B IC $\mathrm{C}_{50}>100 \mu \mathrm{M}$

KDM6

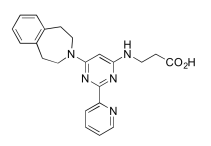

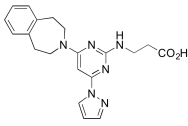

CSK 14.40

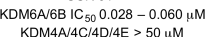
KDM5A IC 506.8 uM KDM5B IC $50.17 \mu \mathrm{M}$ KDM5C IC 50.05 uM
PHF8 IC IC $_{50} 28$ uM Cmpd 19 from 55
KDM6B IC $50.15 \mu \mathrm{M}$ KDM6B IC $50.15 \mu \mathrm{M}$
Unselective

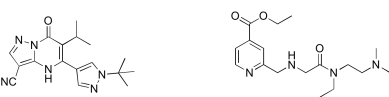

$$
\begin{aligned}
& \begin{array}{cc}
\text { Ex } 117 \text { from } & \begin{array}{c}
\text { Entry } 65 \text { from } \\
\text { WO-2015135094 A1 }
\end{array} \\
\text { Wo-2014053491 A1 }
\end{array}
\end{aligned}
$$

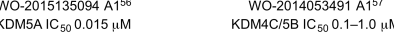

$$
\text { OH }
$$

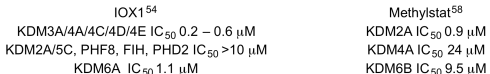

KDM6A IC $\mathrm{C}_{50} 1.1 \mathrm{MM}$
KDMGB IC
$50.14 \mu \mathrm{M}$

KDM6B IC $C_{50} 0.14 \mu \mathrm{MM}$
BBOX $1 \mathrm{C}_{50}>100 \mathrm{\mu M}$ 
FIGURE 4

\section{KDM}

growth factors, cytokines

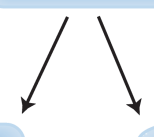

metabolic state

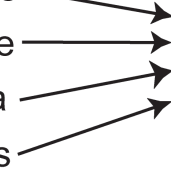

Enzymatic function

Scaffold function - association with chromatin complexes inhibitors
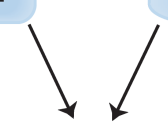

epigenomic changes

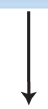

transcriptional regulation 